Supplement of Clim. Past, 15, 827-847, 2019

https://doi.org/10.5194/cp-15-827-2019-supplement

(c) Author(s) 2019. This work is distributed under

the Creative Commons Attribution 4.0 License.

(c) (i)

Supplement of

\title{
Long-term variability of drought indices in the Czech Lands and effects of external forcings and large-scale climate variability modes
}

Jiří Mikšovský et al.

Correspondence to: Jiří Mikšovský (jiri@ miksovsky.info)

The copyright of individual parts of the supplement might differ from the CC BY 4.0 License. 

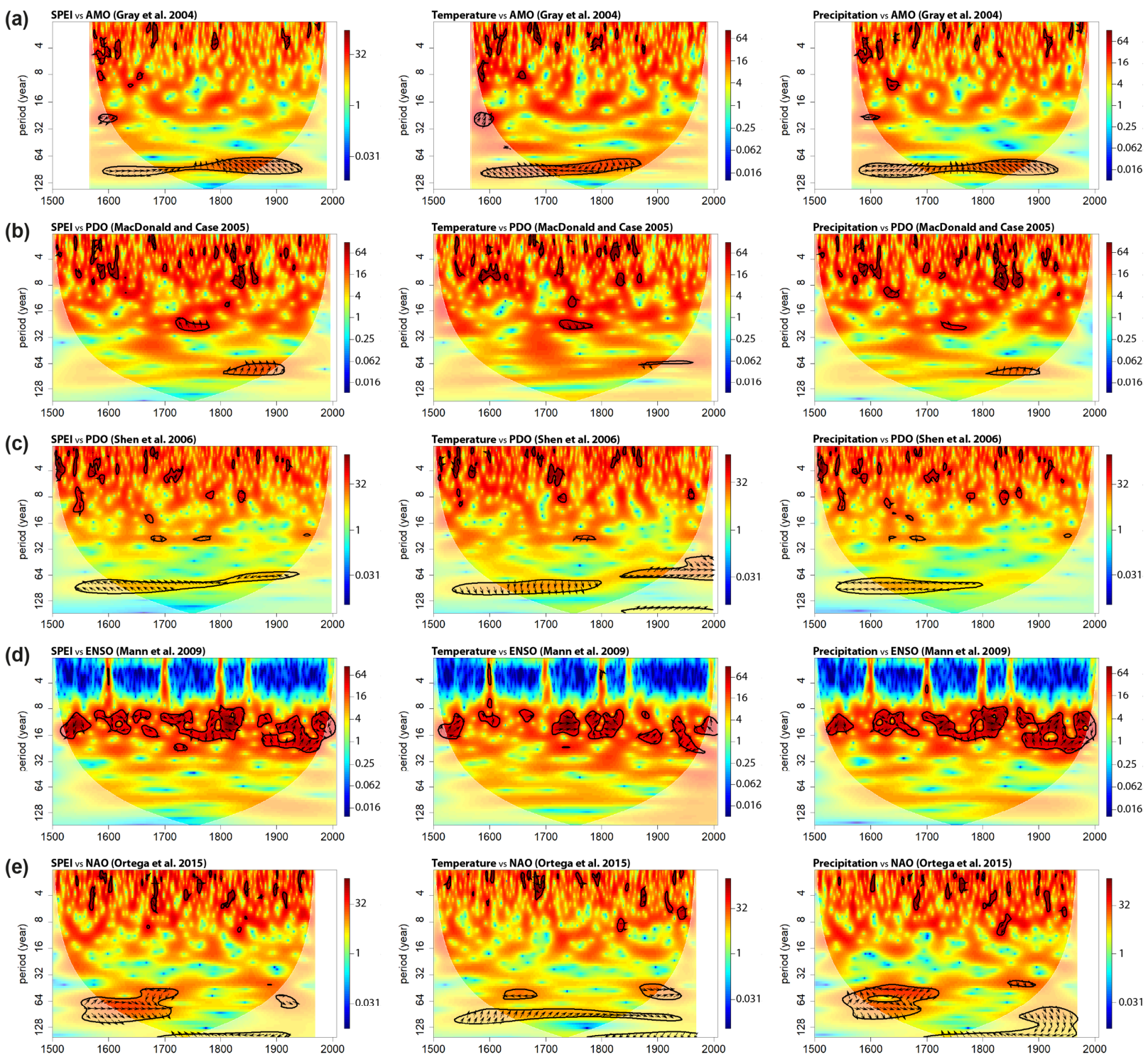

Figure S1. Standardized cross-wavelet power between series of Czech SPEI (left column), central European temperature (central column) and Czech precipitation (right column), and additional explanatory variables with distinct oscillatory component, not included in Fig. 8: (a) AMO reconstruction by Gray et al. (2004), (b) PDO reconstruction by MacDonald and Case (2005), (c) PDO reconstruction by Shen et al. (2006), (d) ENSO reconstruction by Mann et al. (2009), (e) NAO reconstruction by Ortega et al. (2015). Annual time-step; standardized and bias corrected, as per Veleda et al. (2012). Wavelet powers statistically significant at the $95 \%$ level are enclosed by black line; arrows indicate local phase difference, with $\rightarrow$ corresponding to the two signals being in phase and $\leftarrow$ indicating a shift of half the period. The lower-contrast areas pertain to the cone of influence, i.e. a region with diminished representativeness of the wavelet spectra due to edge effects. 

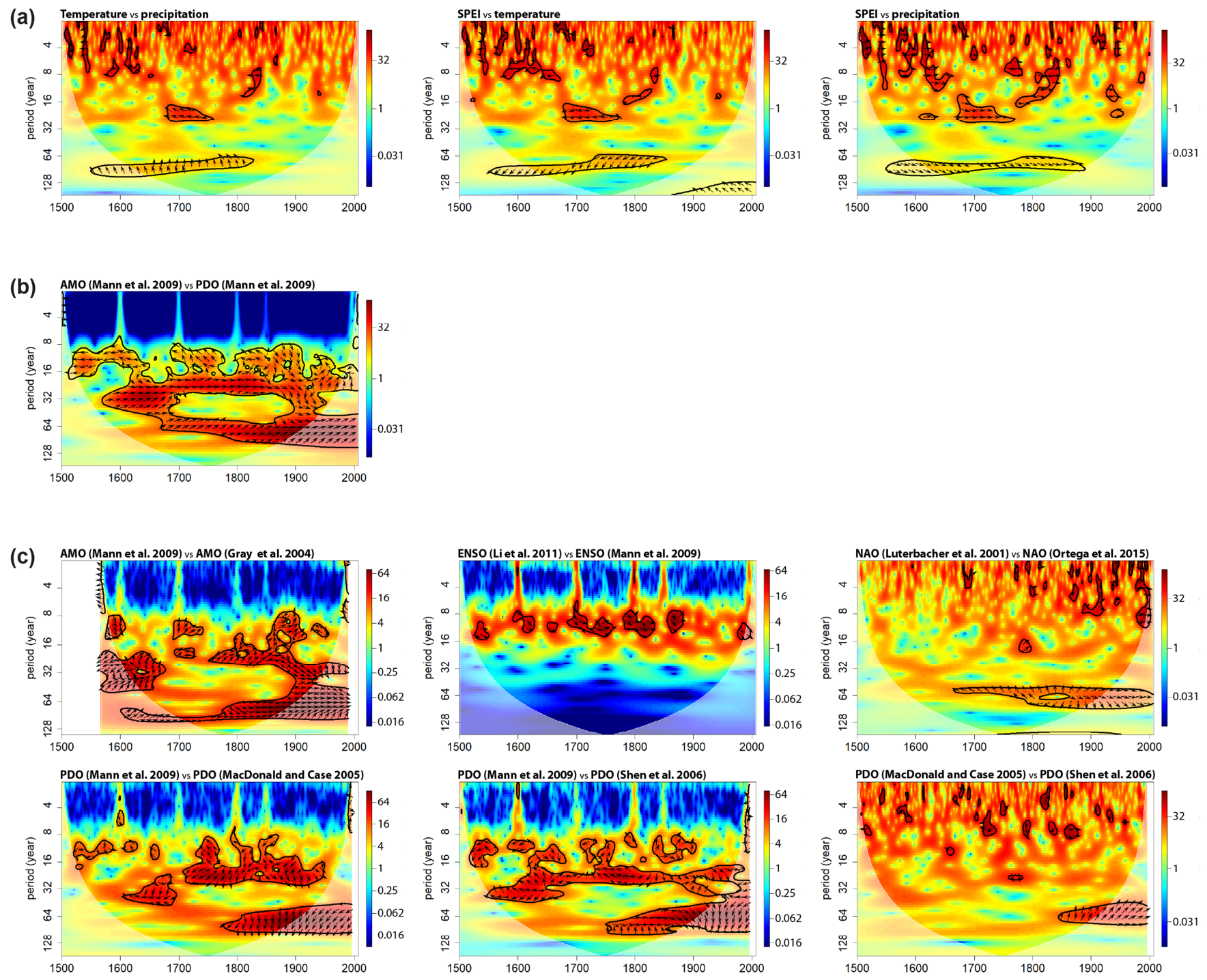

Figure S2. (a) Standardized cross-wavelet spectra between series of Czech SPEI, central European temperature and Czech precipitation.

(b) Standardized cross-wavelet spectra between selected pairs of different explanatory variables. (c) Standardized cross-wavelet spectra between different variants of the same type of explanatory variable. Annual time-step; standardized and bias corrected, as per Veleda et al. (2012). Black line: regions with joint oscillations significant at the 95\% level; arrows indicate local phase difference, with $\rightarrow$ corresponding to the two signals being in phase and $\leftarrow$ indicating a shift of half the period. The lower-contrast areas pertain to the cone of influence, i.e. a region with diminished representativeness of the wavelet spectra due to edge effects. 

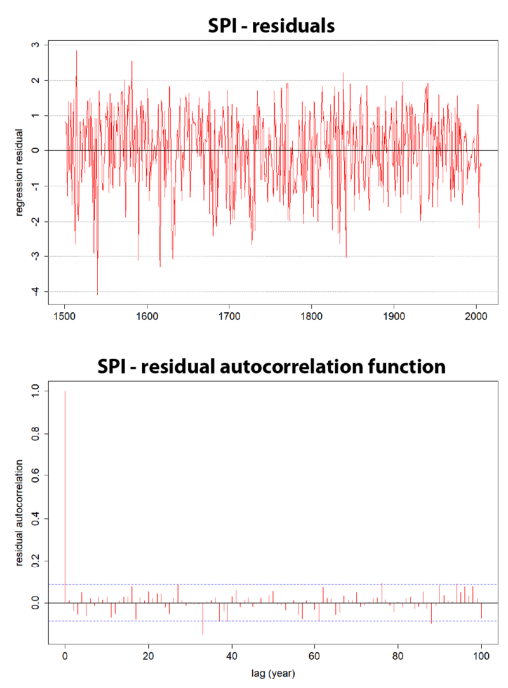
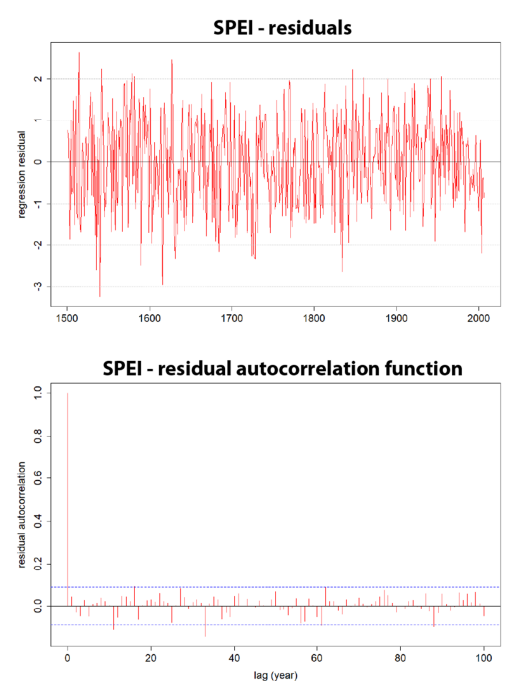

PDSI- residuals

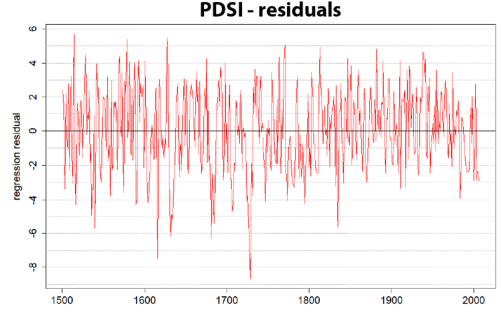

PDSI - residual autocorrelation function

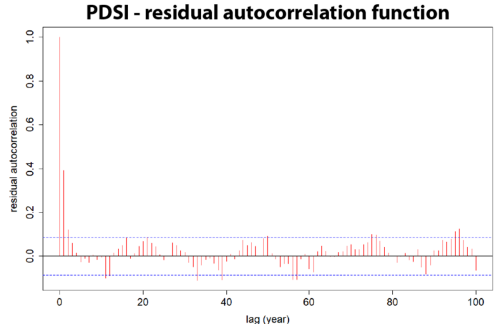

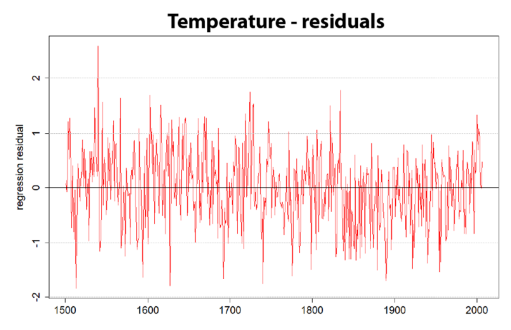
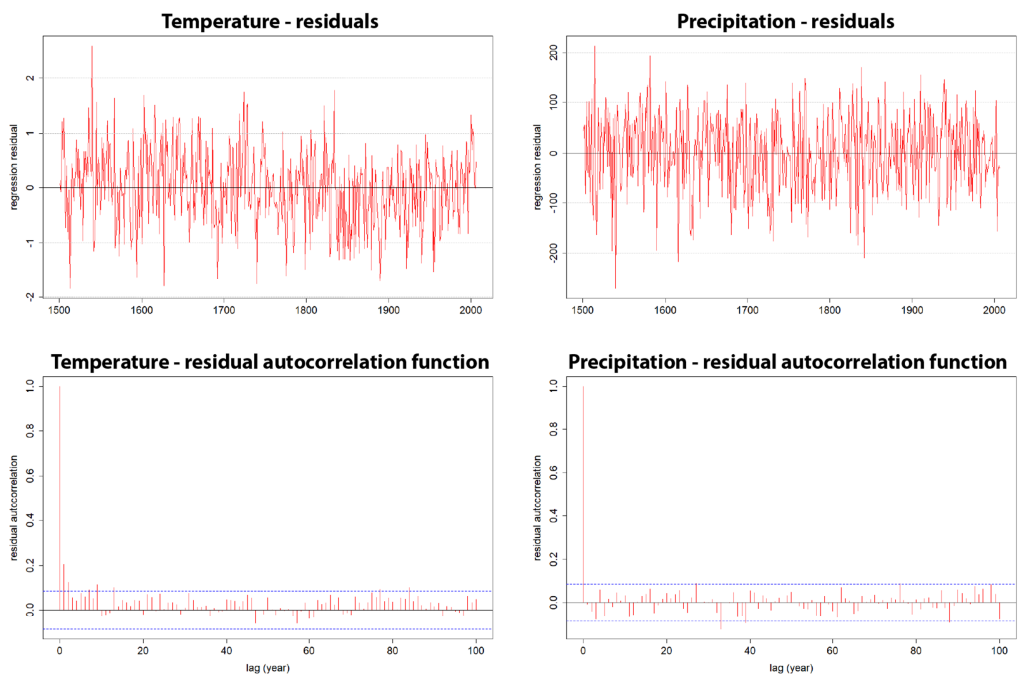

Figure S3. Regression residuals and residual autocorrelation functions, obtained for SPI, SPEI, PDSI, temperature and precipitation from the analysis setup used in Fig. 4a. Blue dashed lines enclose autocorrelation values consistent with the white noise null hypothesis (95\% confidence level). 


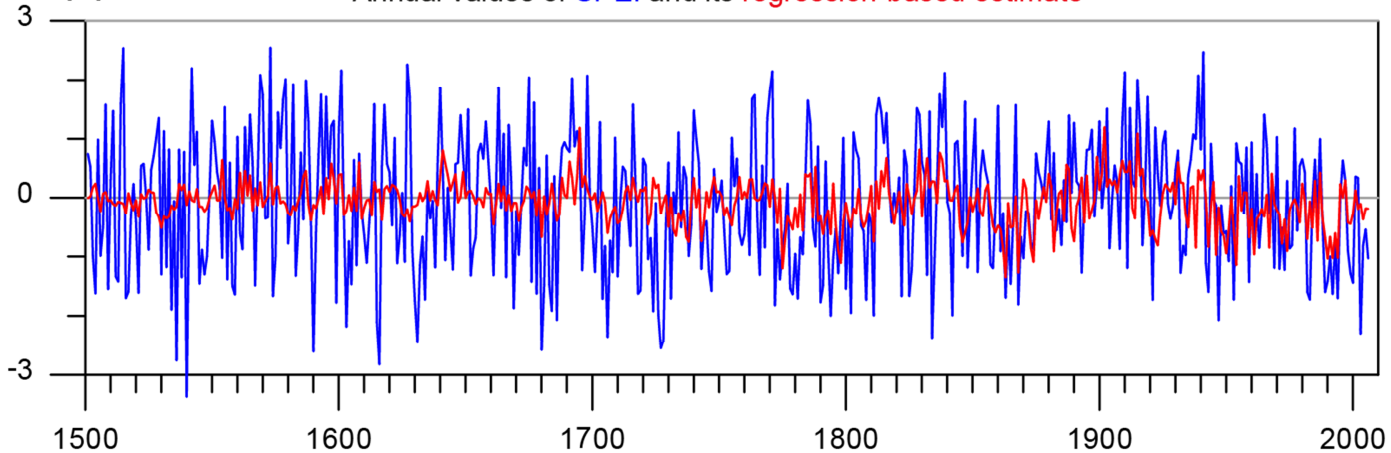

(b) Estimated components of SPEl related to external forcing (GHGs, solar, volcanic)

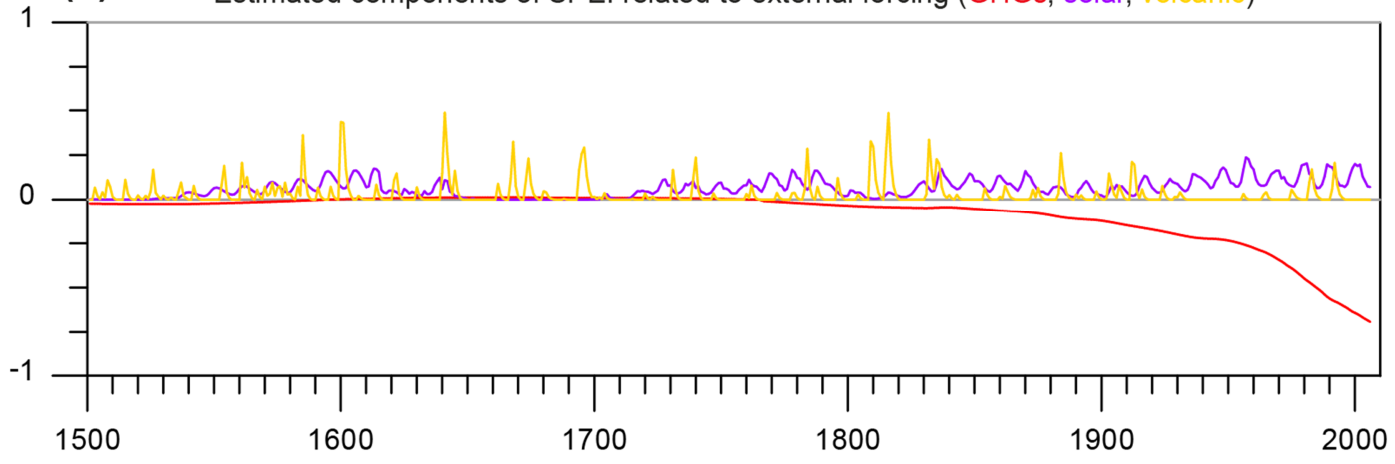

(c) Estimated components of SPEI related to internal variability modes (AMO, PDO, ENSO, NAO)

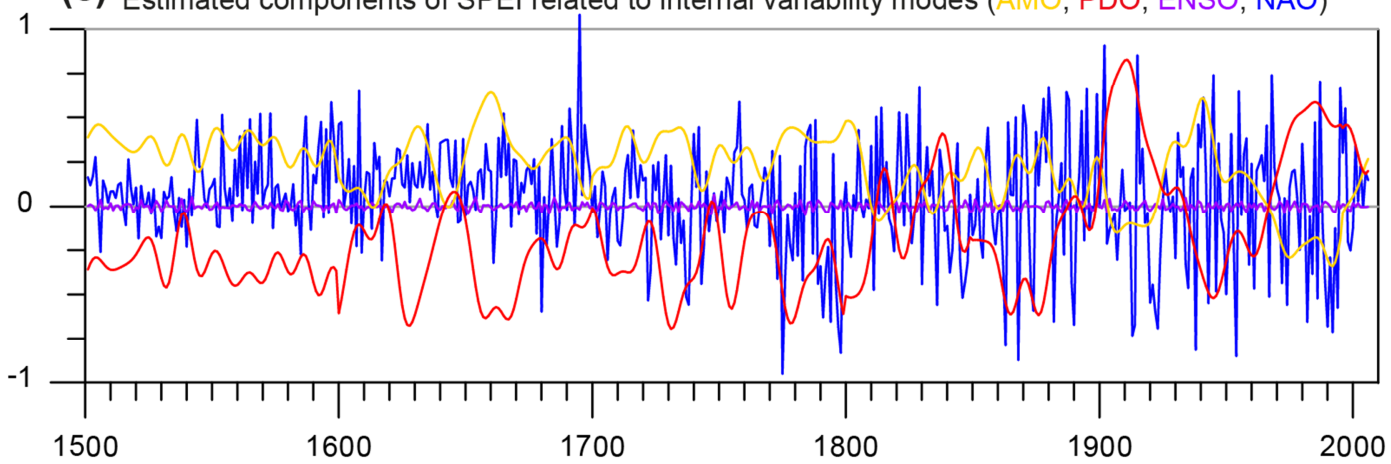

Figure S4. (a) Annual values of SPEI and its regression-based estimate, resulting from the predictor configuration used in Fig. 4a, along with individual regression-estimated responses pertaining to (b) external forcings and (c) internal climate variability modes. 

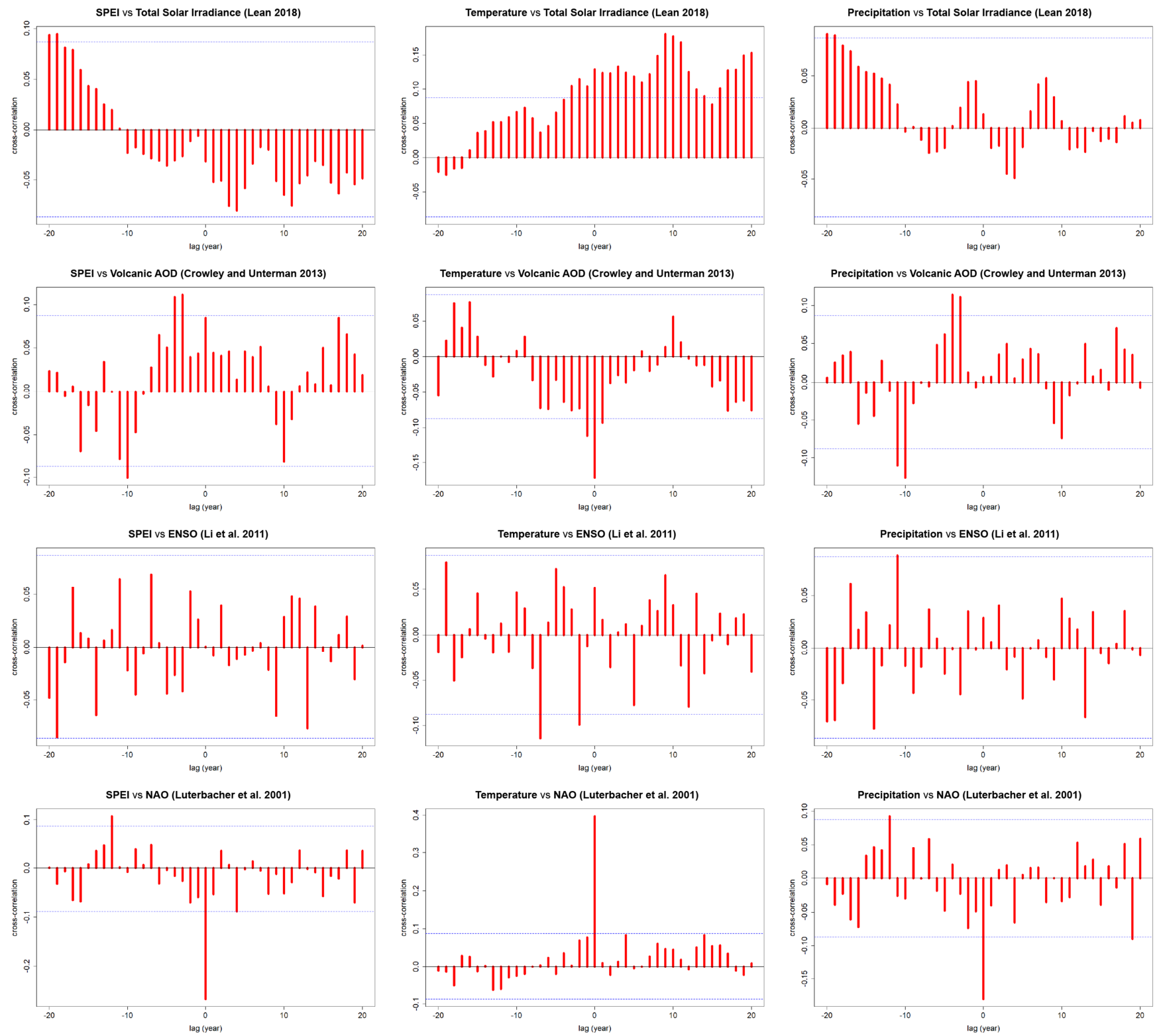

Figure S5. Cross-correlation functions between series of Czech SPEI (left column), central European temperature (central column), and Czech precipitation (right column) and selected individual explanatory variables with pronounced inter-annual variability. Positive values of lag correspond to the first variable being lead by the second. Blue dashed lines enclose correlations consistent with the white noise null hypothesis, at the $95 \%$ confidence level. 


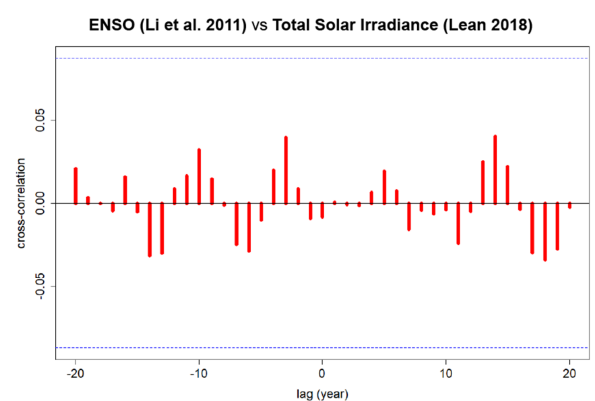

ENSO (Li et al. 2011) vs Volcanic AOD (Crowley and Unterman 2013)

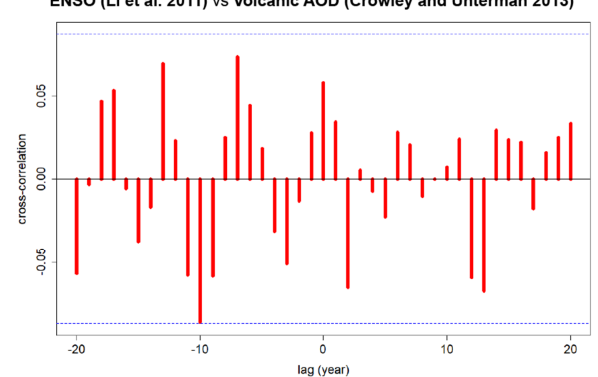

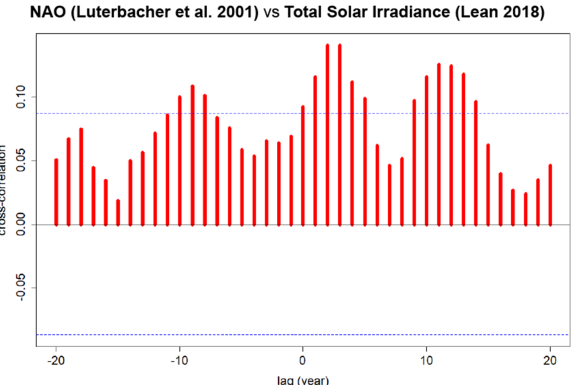

NAO (Luterbacher et al. 2001) vs Volcanic AOD (Crowley and Unterman 2013)

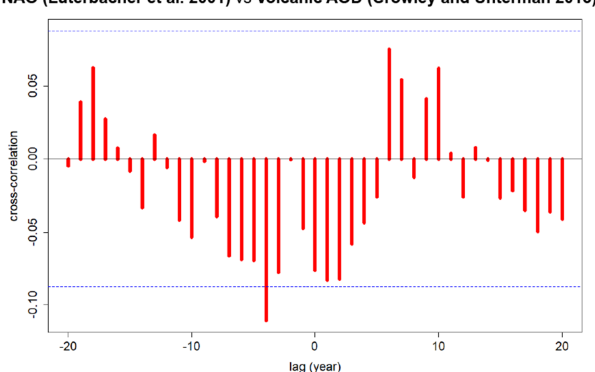

Figure S6. Cross-correlation functions between selected pairs of explanatory variables with pronounced inter-annual variability. Positive values of lag correspond to the first variable being lead by the second. Blue dashed lines enclose correlations consistent with the white noise null hypothesis, at the $95 \%$ confidence level. 